\title{
1
}

\section{Emerging Technologies for Diagnosing Alzheimer's Disease: Innovating with Care}

\section{Marianne Boenink, Harro van Lente, and Ellen Moors}

Public announcements of breakthroughs in diagnosing Alzheimer's disease regularly appear in newspapers, radio and television programmes, and on the web. The types of diagnostic tests recommended range from MRI and PET scans of the brain, to spinal taps, blood tests, simple eye cell tests, and even smelling peanut butter. Most of these tests measure socalled 'biomarkers': certain molecules in the body that are linked with the pathology thought to underlie Alzheimer's disease. The usual claim is that these tests are more reliable, less burdensome, faster and/or cheaper than

M. Boenink $(\bowtie)$

Department of Philosophy, University of Twente, Enschede, The Netherlands

H. van Lente

Department of Technology and Society Studies, Maastricht University, Maastricht, The Netherlands

E. Moors

Copernicus Institute of Sustainable Development, Utrecht University, The Netherlands 
existing diagnostic procedures. But most importantly, the novel tests are thought to reveal Alzheimer's at an early stage, possibly even years before the onset of symptoms.

Early diagnosis of Alzheimer's disease (or AD) is indeed an important, worldwide goal of current research and development in the Alzheimer field (Lock 2013). However, this goal raises controversy, in society, in healthcare, even among those active in Alzheimer research themselves. Proponents argue that an early diagnosis may help to plan one's future life-for example, by deciding whether to continue living in one's own house, by making care arrangements in a timely manner, and possibly, by signing a living will guiding decision making with regard to end of life. Moreover, medication is thought to be possibly more effective when started early. An early diagnosis, followed by early medication, might then help to keep the disease at bay. However, opponents counter that this hope is futile. Current medication slows down the disease, but does not cure it. Early diagnosis and early medication, thus, probably will just extend the time spent on worrying about one's mental capacities. What is the use of an early diagnosis, critics argue, if nothing can be done about the disease? Some even suspect that the whole search for early diagnostics is largely driven by an attempt of 'big pharma' to increase the market for their $\mathrm{AD}$ drugs. Whatever the motives driving R\&D, the response to news items or blogs announcing diagnostic breakthroughs shows that people do indeed hold different views about the desirability of early diagnosis for AD.

\section{The Desirability of Biomarker Diagnostics of Alzheimer's Disease}

From an ethical and societal perspective, the desirability of early diagnostics for $\mathrm{AD}$ is, then, not self-evident. As in other cases of emerging technologies, novel tests for $\mathrm{AD}$ raise the question whether we should do everything we can. Should early diagnostics for AD be introduced in society? This book delves into the issues raised by the promises of early diagnostics for $\mathrm{AD}$ by asking under which conditions emerging diagnostic technologies for $A D$ could be considered a responsible innovation. This question entails more than a reflection on the ethical and/or social acceptability 
of novel tests. In our view, the question whether it is ethically and socially acceptable to introduce early diagnosis for $\mathrm{AD}$ is important, but not sufficient. Three additional questions need to be addressed as well. First, we need to inquire how 'Alzheimer's disease' gets defined in discussions about early diagnostics in the first place, since it refers to an equivocal, poorly delineated phenomenon. Second, it is important to critically assess the plausibility of the promises and expectations about the new diagnostic technologies to avoid speculative ethics. And last but not least, if we are interested in the ethical and social acceptability of these emerging technologies, we should not only identify and weigh social and ethical values, but we should also examine the capacity of contemporary society to productively respond to the diversity of viewpoints, concerns, and interests voiced with regard to these technologies. We will briefly discuss these three questions, and then, return to the ambition of responsible innovation.

According to historians and philosophers of medicine, the phenomenon of $\mathrm{AD}$ is notoriously elusive. The German psychiatrist Alois Alzheimer, in 1906, during an autopsy, identified 'plaques and tangles' in the brains of a patient who had suffered from what was then known as 'senile dementia' at a relatively young age. Whereas the plaques had been seen before, the tangles were a new phenomenon. It was actually Alzheimer's boss, Emil Kraepelin, who-in the 1910 edition of his famous classification of psychiatric diseases - coined the term 'Alzheimer's disease'. AD was defined as a specific form of dementia, diagnosed in the case of a young age of onset of the dementia symptoms and when, at autopsy, both plaques and tangles were found. (The presence of plaques and tangles has been part of the gold standard for diagnosing $\mathrm{AD}$ ever since.) The disease has been distinguished from other forms of dementia, that is, by its pathological features. Since its inception, however, the assumed relation between clinical features and pathological signs of the disease has been shifting time and again. As the historian Jesse Ballenger (2006) has shown, both the definition of the clinical picture and of the pathology of $\mathrm{AD}$ have evolved. Even more importantly, whatever the definitions used, the relation between clinical and pathological phenomena has never been unambiguous. Plaques and tangles can be absent in persons clinically diagnosed with $\mathrm{AD}$, whereas they may be present in the brain of people who did not experience any trouble during their lifetime. 
Clarifying the relationship between clinical features and pathology is complicated, for various reasons. First, usually, there is a time lag between clinical observations (during life) and pathological observations (at autopsy). During life, we can only see the dementia symptoms, not the $A D$ pathology. Part of the promise of current research is that molecular biomarkers will help to overcome this lag, because they can show pathology in vivo. However, ageing is a confounding influence. Are the clinical and pathological observations characteristic of 'normal ageing' or of a disease? Moreover, the clinical symptoms of AD are various and not very specific. They may signify others types of dementia. And in particular, older patients often suffer from other diseases (they have 'co-morbidity'). To what extent current candidate biomarkers are specific for $\mathrm{AD}$ is as yet unclear. All this variety has brought some researchers to the conclusion that $\mathrm{AD}$ should not be seen as a unified disease, but as a diffuse syndrome of several phenomena (Richards and Brayne 2010; Richard et al. 2012). These phenomena, moreover, are not just present or absent, but can show different grades of severity. This goes both for the clinical and the pathological manifestations. In sum, suggesting that biomarker testing can reveal $A D$ is a vague claim, to say the least. Without further clarification, such claims ignore the ambiguity of the label and the complexity of the associated phenomena. Since assessing the desirability of diagnosing AD is impossible if we do not know what is being diagnosed, this volume will pay ample attention to the different meanings of the AD label in different contexts. This is also the reason we do not limit attention to $\mathrm{AD}$, but focus on emerging $\mathrm{AD}$ diagnostics in the broader context of diagnosing and dealing with dementia.

The second task is to critically assess the promises or expectations about how easy, convenient, early, and reliable diagnosing $\mathrm{AD}$ will be in the future, due to new technologies. Clearly, clarifying the meaning of $\mathrm{AD}$ in such claims is a first step, as well as asking what exactly is made visible by the new technology, and what this tells about the prospects of the individuals tested. The rhetoric of breakthroughs and revolutions is typical for emerging science and technology in general, but the field of $\mathrm{AD}$ research seems particularly prone to it. $\mathrm{AD}$ is perceived by many as an awful disease that they dread, and this anxiety is reinforced by predictions about rising numbers of $\mathrm{AD}$ patients in the near future-frequently 
expressed in terms of an Alzheimer 'tsunami' or an 'epidemic'. With the awareness that decades of $R \& D$ have not resulted in an effective cure, any positive news from the $R \& D$ trenches is easily framed (by researchers, media, politics, policymakers, and public alike) as a reason for optimism and hope. For discussions about the desirability of early diagnosis, however, it is crucial to determine what these promises and expectations are actually based on. If a targeted biomarker is tested only in mice, it may be rather premature to claim that early diagnosis is near. In a similar vein, if the candidate biomarker is considered to be a predecessor of the plaques associated with $\mathrm{AD}$, what does that mean for people suffering from complaints, but not displaying plaques and tangles? And will biomarker diagnostics be a 'stand-alone' test offering a yes/no verdict, as often suggested, or will it rather be an 'add on' to the existing diagnostic repertoire? Assessing the plausibility of the promises and expectations raised on behalf of emerging diagnostic technologies helps to avoid what has been called 'speculative ethics' (Nordmann 2007; Nordmann and Rip 2009; Lucivero et al. 2011). It is a prerequisite for down-to-earth reflection and debate on the ethical and societal desirability of emerging biomarker tools.

Third, asking about the ethical and social acceptability of introducing emerging technologies for diagnosing $\mathrm{AD}$ suggests that after weighing the pros and cons, only two answers are possible: yes or no; end of story. Moreover, the implicit assumption is that society can —and will-act on such an ethical verdict, as if there is a central gatekeeper determining whether the technology should be allowed. This seems an overestimation of both the willingness and the ability of current societies to steer innovation, or, if you prefer, an underestimation of the complexity of innovation processes. It is not very likely, for example, that contemporary governments will forbid industry from pursuing specific goals in R\&D, unless there are serious concerns to health, environment, and safety. Diagnostic test providers can also easily avoid self-regulation by doctors (e.g., in the form of clinical guidelines for diagnosing AD) by offering direct-toconsumer-testing via the internet. More importantly, aiming for a yes/no verdict neglects opportunities for shaping innovation processes and their products in a more desirable direction. It may result in an unproductive sequence of emerging innovations and ethical or societal rejection of such 
innovations. Asking about conditions for responsible innovation allows us to bring into focus ways of shaping emerging technologies to align with society and its values, and at the same time to identify the actors (or actor groups) responsible for doing so.

\section{Responsible Innovation}

Our choice to reflect on the desirability of emerging biomarker diagnostics for $\mathrm{AD}$ in terms of responsible innovation is in line with (and a product of) a growing interest in 'responsible innovation' more generally. The notion of responsible research and innovation (for reasons of brevity, from now on, referred to as 'responsible innovation') has recently emerged as a guiding concept in discussions about the science-society relationship_-in particular in Europe and to a lesser extent in the USA. It is rooted in the observation that scientific and technological advances not only produce benefits, but may have unintended and undesirable impacts, and that regulating the products of these advances (e.g., by requiring risk assessment) is insufficient, and sometimes, impossible because of the uncertainties involved. By aiming for 'responsible innovation', attention is sought not only for the potential negative impacts of innovation, but also for the positive ones. To achieve an overall positive result, both the process and the products of scientific research, technology development, and implementation should be designed in such a way that they contribute to relevant and acceptable societal goals. To make science and technology align better with society, its values should be integrated into the full innovation trajectory. Finally, the concept of responsible innovation explicitly puts on the agenda the question who, in the largely collective and complex endeavour of innovation, should take care of what to work towards relevant and acceptable benefits.

The notion of responsible innovation, thus, refers to an overarching concern and a set of partly overlapping approaches and concomitant definitions. Currently, two definitions and frameworks are widely cited. The first is by Von Schomberg:

Responsible Research and Innovation is a transparent, interactive process by which societal actors and innovators become mutually responsive to 
each other with a view to the (ethical) acceptability, sustainability and societal desirability of the innovation process and its marketable products (in order to allow a proper embedding of scientific and technological advances in our society). (Von Schomberg 2013, p. 63)

In this definition, responsible research and innovation designates the search for the right impacts of science and technology. Von Schomberg observes that shared criteria to determine what these 'right impacts' are, are not easy to identify in current pluralistic societies. However, he argues that the values democratically agreed upon in the Treaty of the European Union might serve as normative anchor points to decide what is ethically acceptable and socially desirable. These include scientific and technological advance, sustainable development, competitive social market economy, social justice, equality, solidarity, fundamental rights, and a high level of quality of life. As the definition indicates, both the process and the products of innovation need to be assessed in terms of these anchor points to ensure responsible research and innovation.

Von Schomberg developed his take on responsible research and innovation in the context of European research funding and research policymaking, and his approach addresses this level of policymaking in the first place. Some scholars have argued that these rather abstract principles offer less guidance for specific R\&D projects (Stilgoe et al. 2013, p. 1577). It is, for instance, not clear how to identify which principles are at stake in a specific setting, nor how to interpret their meaning when it comes to decisions in a specific innovation trajectory, or how to balance them. The approach of responsible innovation proposed by Stilgoe, Owen, and Macnaghten is, therefore, more concerned with particular domains in science and technology. It was developed on the basis of an inventory of concerns recurring in public debates about new domains of science and technology. These target the products, the process, and the purpose of innovation, and responsible innovation in this approach is a way to embed deliberation on these issues in the innovation process. Stilgoe and colleagues define responsible innovation as follows:

Responsible Innovation means taking care of the future through collective stewardship of science and innovation in the present. (Stilgoe et al. 2013, p. 1570) 
This definition is rather open and does not refer to specific normative ideals, but to a caring, future-oriented attitude. Stilgoe and colleagues propose a framework of four integrated dimensions that might be helpful for guiding responsible innovation on the level of innovation governance, and also within $\mathrm{R} \& \mathrm{D}$ projects. Responsible innovation, in their view, consists of anticipation (systematic thinking about future developments and the uncertainties implied), reflexivity (both individual and institutional), inclusion of stakeholders to increase the public and moral legitimacy of decisions and outcomes of innovation (later labelled as 'deliberation', see Owen et al. 2013), and finally, responsiveness: a 'capacity to change shape or direction in response to stakeholder and public values and changing circumstances' (Stilgoe et al. 2013, p. 1572).

\section{Innovating with Care}

The authors in this volume explore what it would mean to innovate responsibly in the domain of emerging technologies for diagnosing $\mathrm{AD}$. In doing so, we aim to contribute not only to societal and policy debates about emerging diagnostics for $\mathrm{AD}$, but also to academic and policy discussions about responsible innovation more generally. Obviously, interpreting the concept of responsible innovation for a specific domain such as $\mathrm{AD}$ diagnostics is not a straightforward task. As outlined above, it makes sense to conceive of responsible innovation as a set of interdependent and partly overlapping activities: anticipation of the meaning and potential impacts of early diagnostics, exploring the views and values of potential stakeholders, and enhancing the reflexivity as well as the responsiveness of the actors involved. Together, these activities contribute to an innovation process that takes into account the shared values emerging in this process. However, this starting point leaves ample room for different interpretations and specifications in practice. For a start, the relative importance and the order of the activities mentioned may differ. More subtle differences result from the way the activities are performed. How exactly should one go about stimulating anticipation, reflection, deliberation, and responsiveness in a specific setting? It is hard to briefly account for the choices made in this volume, but in general our approach has been guided by the desire to avoid two pitfalls in particular. 
The first pitfall is to frame an envisioned innovation and its eventual impacts exclusively as a future concern. The concern with emerging, uncertain developments and their even more uncertain impacts makes responsible innovation discourse prone to looking forward only. In our view, this would downplay the importance of the present situation. Innovation is not introduced into a void; it transforms the world as we know it and the values realized in that world. Nor do the changes start only after a new technology has been introduced; promises and expectations raised may have an impact already on existing practices. To assess under which conditions emerging diagnostics of $\mathrm{AD}$ is desirable, then, we will pay careful attention to the present. what is it that might be improved, shifted, or lost by this innovation? What exactly are the positives (and negatives) realized in current practices of diagnosing $\mathrm{AD}$ ? And where and how are actors working on improving these practices? Evaluating how (promises about) an innovation might affect diagnostic practices implies that we know what is at stake in those practices, but also, which other attempts to innovate them are ongoing.

The second pitfall is to assume that current values are easily accessible. As outlined above, current approaches of responsible innovation suggest that values can be identified either by looking for principles that have been explicitly agreed upon (such as those in the EU treaty), or by asking stakeholders to voice what is important to them. Both methods imply that values are given, discursive entities, which can be made explicit when necessary. However, as research from pragmatic ethics (Dewey 1902a, b; Keulartz et al. 2004), care ethics (Tronto 1993; Pols 2012, 2014), science and technology studies (Mol et al. 2010), and empirical philosophy of technology (Verbeek 2011) has made abundantly clear, values are embedded in human practices and are realized in ways of doing good, by using specific routines, concepts, and materials. If we separate values from their practical embedding, we risk a seriously impoverished view of what is at stake. When exploring the values strived for and realized in practice, then, we will make ample use of ethnographic and analytical methods, rather than asking stakeholders right away. The results of these explorations may not only inform, but also elicit subsequent stakeholder reflection and deliberation.

Overall, then, we will tackle the question of responsible innovation of biomarker diagnostics for $\mathrm{AD}$ in a thoroughly practice-based way. We 
aim to do justice to the complex and intricate relations between values, interpretative frameworks, and social and material practices, both in the present and in the future, opened up by emerging technologies. Innovating responsibly, in our view, means that the richness and complexity of both current and potential future practices are acknowledged and handled with care. If we want emerging technologies for diagnosing $\mathrm{AD}$ to truly improve current practices of dealing with $\mathrm{AD}$, we have to explore: (1) how current practices of diagnosing and living with $\mathrm{AD}$ imply specific views of the world and of what is (or is not) valuable, (2) how the aimed-for innovation(s) might alter these practices, and therewith, the complex web of de facto values, and (3) how innovation and practice can be aligned with each other in such a way that the result can be considered an improvement. We have dubbed our approach 'innovating with care', to emphasize that to be responsible, innovation should proceed in a perceptive and careful way.

While we do not propose a full-fledged, generic methodology or framework for responsible innovation, we believe that the ideas guiding our approach of innovating with care are relevant for other cases and fields of innovation as well. Our approach guides thinking about particular innovations and R\&D projects by directing the way anticipation, reflection, inclusion/deliberation, and responsiveness are handled: by paying careful attention to the present as well as the future, and to the complex interrelations between values, interpretative frameworks, and social and material practices. This may be particularly useful for settings where, as in the $\mathrm{AD}$ field, ample controversy exists with regard to what 'good practice' is.

\section{Chapter Outline}

This volume provides, then, observations, analyses and reflections that may inform any attempt to innovate with care in the field of AD diagnostics. It will not pass final, overall judgement on the desirability of such diagnostics, but it will point out the conditions to innovate responsibly in this specific area. Moreover, we will reflect on what this implies for responsible innovation in diagnostics, more generally. The book is divided into four parts and a concluding chapter. 
Part I (Biomedical Research on AD Diagnostics: Background and Trends) reconstructs and analyses developments in biomedical research on the diagnosis of $\mathrm{AD}$ and dementia. It sketches how the field has evolved into the current state of affairs and discusses important visions, concepts, and research practices on $\mathrm{AD}$ and dementia. This sets the stage for a broader assessment of these developments in terms of 'responsible innovation' in the subsequent parts.

Peter Whitehouse (Chap. 2) outlines the historical developments in biomedical research on AD and dementia. He presents the developments in pharmaceutical research(in which he was personally involved) and discusses how the limitations of present drugs for AD are at the background of the recent attempts to diagnose $\mathrm{AD}$ at an earlier stage. $\mathrm{He}$ then explains why such research can be labelled 'irresponsible' because it suggests that earlier diagnosis is always for the better, and because it raises false hope with regard to the possibility of a (biomedical) cure. This shot across the bows is followed by a more elaborate discussion of the current state of affairs in biomedical $\mathrm{AD}$ research in the following chapters.

Annette Leibing (Chap. 3) explores the recent trend towards AD prevention. She reconstructs how the emergence of a cardiovascular logic, in combination with the emerging possibility of detecting presymptomatic biomarkers, has considerably impacted the conceptual frameworks used to interpret and deal with AD. An important question now is whether preventive interventions should focus on lifestyle, drugs, or both, and who has access to such interventions. In view of the complexities involved, she argues, both biomedical researchers and social science or humanities scholars should make an effort to distinguish short cuts from valid research.

Marianne Boenink (Chap. 4) analyses how current research to identify molecular biomarkers for $\mathrm{AD}$ strives to link biological observations with patient needs. To this end, she analyses how the interrelation of biological and clinical phenomena related to $\mathrm{AD}$ is conceptualized, pursued, and shaped in: (1) discourse on the future of medicine, (2) scientific literature on AD biomarkers and new guidelines for diagnosing the disease, and (3) the design of a specific biomarker research project. Since the aimed-for connections are rather elusive, biomarker research in practice easily slides into basic molecular biology research. She suggests 
that strengthening both 'epistemic' and 'translational' responsibility in $\mathrm{AD}$ research might help to increase its relevance and usefulness for those who suffer from AD.

In Chap. 5, Richard Milne and Shirlene Badger examine a different strand of current $\mathrm{AD}$ research: the emergence of 'big data' initiatives and the related trend to re-purpose existing observational cohort studies of ageing populations. Current cohort research not only studies the development of AD and dementia, but also aims to create a source of participants for intervention trials. Milne and Badger argue that to give meaning to the idea of responsible research and innovation, more attention should be paid to the research practice of cohort studies and the establishment of responsive relations between researchers and participants. In particular, the role of interviewers, study coordinators, and research nurses in such studies offers valuable opportunities to truly care for the needs of research participants.

In Part II (Diagnosing Alzheimer's Disease: Current Practices), we delve into current practices of diagnosing $\mathrm{AD}$ and dementia, with a particular focus on the values embedded in these practices. As argued above, a good understanding of current practices is crucial to anticipate how emerging biomarker technologies may interact with these practices, and to reflect on what this would mean for the realization of values. Most of the hopeful discourse surrounding emerging $\mathrm{AD}$ diagnostics presupposes that current practice of diagnosing $\mathrm{AD}$ is (a) uniform and (b) deficient, because it is complex, time-consuming, and produces unreliable results. AD diagnostics is thought to produce a lot of uncertainty, both for the people diagnosed, their families, and the professionals involved-uncertainties that the emerging diagnostic tools are expected to resolve. In contrast, the authors in Part II highlight the plurality and complexity of current practices of diagnosing AD. They also show how $\mathrm{AD}$ diagnostics is evolving anyway, partly because of innovations outside the biomedical domain. As a result of this complexity and ongoing changes, the proposed introduction of biomarker tools is likely to have different impacts in different locations. Moreover, the problems innovations of diagnostics claim to solve need not be shared by all diagnostic practices alike.

Anna Laura van der Laan (Chap. 6) observes that current medical practice of diagnosing $\mathrm{AD}$ and dementia in the Netherlands is very 
heterogeneous. She distinguishes two general modes of diagnosing: 'pulling out all the stops' and 'holding back', and shows how these imply different sets of values. In addition, she reconstructs how the differences are distributed in practice: either on 'factual' grounds (sorting patients into specific disease subcategories), or on normative grounds (patients' preferences). Both lead to problems and uncertainties, however, because facts and values in the area of AD diagnosis are intricately related. Van der Laan concludes that emerging biomarker tools may improve this situation, provided they contribute to taxonomies that actually 'matter' to people.

Julia Swallow (Chap. 7) explores the role of low-technological cognitive screening tools in the process of diagnosing $\mathrm{AD}$ in everyday clinical practice in the UK. She reconstructs how the tools emerge as provisional, yet privileged devices for navigating uncertainty through the tinkering work of clinicians. However, as the tools are adopted in frameworks promoting early diagnosis, such as the National Dementia Commissioning for Quality and Innovation Framework (CQUIN), this tinkering work is constrained. Swallow concludes that when developing high-tech diagnostic tools, the adaptive and uncertainty-navigating strengths of low-tech screening tools should not be overlooked. Moreover, since such high-tech diagnostic innovation is likely to bring along further uncertainties and controversies, responsible innovation in this area should ensure possibilities for tinkering diagnostics according to the circumstances.

In Chap. 8, Claudia Egher and Sally Wyatt point out that AD diagnostics is not just taking place in the doctor's consultation room, but is available on the internet as well. This chapter starts from the assumption that the internet is an innovative diagnostic technology, and explores how digital technologies in all their multiplicity are affecting interactions and processes associated with diagnosing $\mathrm{AD}$. It focuses particularly on how responsibilities in diagnosis are shifting already.

Part III (Alzheimer's Disease: Multiple Realities and Concerns) broadens the scope of discussion by focusing on the practices and meanings $\mathrm{AD}$ and dementia have beyond the biomedical domain. The authors contributing to this part explore the multiple meanings and interpretative frameworks' surrounding $\mathrm{AD}$, dementia, and early diagnosis in a wide array of locations and practices. They also examine how current promises and expectations of biomarker technologies and early diagnostics relate 
to those practices. As in the preceding part, the recurring question is what the multiplicity of meanings and interpretations implies for responsible innovation of $\mathrm{AD}$ diagnostics.

Ingunn Moser (Chap. 9) explores how Alzheimer's disease is being shaped as a 'matter of concern' in a number of locations, including: the international Alzheimer's patients' movement; medical textbook and diagnostic context; laboratory science; daily care practice; an advertisement for anti-dementia medication; general practice; and parliamentary politics. She makes visible how all these practices are implicated in politics by framing the reality of and concerns with $\mathrm{AD}$ in a specific way and by interfering with alternative framings. Making visible how current practices concerning $\mathrm{AD}$ are implicated in politics, she argues, is crucial to make these politics more open, reflexive, and collective, thus contributing to responsible innovation.

In Chap. 10, Yvonne Cuijpers discusses the worldwide emergence of national dementia strategies. Since multiple approaches to dementia coexist, strategies to address dementia as a nation are not straightforward. Cuijpers provides a reconstruction and analysis of which framings of dementia are articulated in the course of the development of a Dutch dementia strategy, and how stakeholders deal with the coexistence of multiple framings of dementia. The chapter delineates three models of coexistence: a model where different frames are considered 'fragments of a whole'; one where they are in antagonistic positions; and a model where different frames move in different directions. The process of constructing a national dementia strategy and the surrounding discussions take different shapes, depending on the assumed model of how approaches to dementia coexist.

Jeannette Pols and Amade M'charek (Chap. 11) take issue with the linear view of innovation that seems implied in discourse on responsible innovation. Focusing on the case of Alzheimer diagnostics, Pols and M'charek demonstrate that innovations do not emerge in such a linear way. Patient advocacy movements engage with scientific research, and research and clinical practices are highly intertwined. Yet, research and clinical practices may also have very different problem definitions, aims, knowledge, concerns, and pace. Pols and M'charek argue that responsible innovation, rather than privileging a particular type of labora- 
tory research, should start innovations by taking notice of the different manifestations of 'Alzheimer problems' and the different science-clinic representation practices needed to address these problems.

In Part IV (Assessing Diagnostic Innovations), we shift focus from current practices of diagnosing and living with $\mathrm{AD}$ and dementia, to practices of assessing diagnostic innovations, in general. In the biomedical domain, extensive assessment and evaluation procedures have evolved since World War II. This part explores the possibilities and limitations of current procedures for Health Technology Assessment in the domain of emerging diagnostics. Moreover, it outlines several suggestions to redesign common health technology assessment procedures to better fit actual innovation practices as well as societal concerns about innovation.

Fiona Miller, Robin Hayeems, and Stuart Hogarth (Chap. 12), drawing on observations of a feasibility study of personalized cancer care, highlight the informally regulated nature of diagnostic innovation systems. Translational imperatives blur clinical and research aims, key regulatory institutions are bypassed, and other cognitive, normative, and regulative institutions encourage attention to test performance, rather than patient outcomes. In these socio-technical systems of limited accountability, intentions to help patients and act ethically are not critically assessed; instead, assumed benefits and a perceived duty to do good tend to produce diagnostic innovations of questionable value. They argue that wider patient and public engagement, together with robust, legitimate, and accountable regulatory regimes will be required to truly innovate with care'.

In Chap. 13, Ellen Moors and Alexander Peine observe that diagnostic innovation is increasingly perceived as an institutional interplay with many heterogeneous stakeholders, in which users are more proactively involved in diagnosis. This challenges traditional Health Technology Assessment (HTA) practices, which usually focus on efficacy, safety, quality, and costs. This chapter zooms in on the current 'logic of valuing' in HTA, and explores several examples of diagnostic innovation to point out the shortcomings of this logic. Moors and Peine conclude that to be responsible, HTA strategies and policies had better take into account the creative and transformative character of innovation. This particularly implies that HTA procedures should allow for more flexibility to respond 
to changes in the actors, values, roles, or responsibilities actually at stake in diagnostic innovation.

In the Conclusion, the insights and observations from the previous chapters are brought together, asking what they mean for the case of emerging technologies for diagnosing AD. What lessons can be learned from our wide-ranging explorations? Which conditions need to be met, which pitfalls avoided, if we aim to innovate AD diagnostics? Here, we also consider the fruits of our practice-based approach for the project of responsible innovation at large: how to innovate with care.

\section{References}

Ballenger, J.F. 2006. Self, Senility and Alzheimer's Disease in Modern America. Baltimore: The Johns Hopkins University Press.

Dewey, J. 1902a. The Evolutionary Method as Applied to Morality I: Its Scientific Necessity. The Philosophical Review 11(2): 107-124.

Dewey, J. 1902b. The Evolutionary Method as Applied to Morality II: Its Significance for Conduct. The Philosophical Review 11(4): 353-371.

Keulartz, J., M. Schermer, M. Korthals, and T. Swierstra. 2004. Ethics in a Technological Culture. A Programmatic Proposal for a Pragmatist Approach. Science, Technology and Human Values 29(1): 3-29.

Lock, M. 2013. The Alzheimer Conundrum. Entanglements of Dementia and Ageing. Princeton: Princeton University Press.

Lucivero, F., T. Swierstra, and M. Boenink. 2011. Assessing Expectations: Towards a Toolbox for an Ethics of Emerging Technologies. NanoEthics 5(2): 129-141.

Mol, A., I. Moser, and J. Pols. 2010. Care in Practice. On Tinkering in Clinics, Homes and Farms. Bielefeld: Transcript Verlag.

Nordmann, A. 2007. If and Then: A Critique of Speculative NanoEthics. NanoEthics 1(1): 31-46.

Nordmann, A. and A. Rip (2009). Mind the gap revisited. Nature Nanotechnology 4(5): 273-274.

Owen, R., et al. 2013. A Framework for Responsible Innovation. In Responsible Innovation, eds. R. Owen, J. Bessant, and M. Heintz, 27-50. Chichester: Wiley. 
Pols, J. 2012. Care at a Distance. On the Closeness of Technology. Amsterdam: Amsterdam University Press.

Pols, J. 2014. Radical Relationality: Epistemology in Care and Care Ethics for Research. In Moral Boundaries Redrawn: The Significance of Joan Tronto's Argument for Political Theory, Professional Ethics, and Care as Practice, eds. G. Olthuis, H. Kohlen, and J. Heier, 175-194. Leuven: Peeters.

Richard, E., B. Schmand, P. Eikelenboom, R.G.Westendorp, and W.A. van Gool. 2012. The Alzheimer Myth and Biomarker Research in Dementia. Journal of Alzheimer's Disease 31(supplement 3): s203-s209.

Richards, M., and C. Brayne. 2010. What Do We Mean by Alzheimer's Disease? British Medical Journal 341: 865-867.

Stilgoe, J., R. Owen, and P. Macnaghten. 2013. Developing a Framework for Responsible Innovation. Research Policy 42: 1568-1580.

Tronto, J. 1993. Moral Boundaries. A Political Argument for an Ethic of Care. New York: Routledge.

Verbeek, P.P. 2011. Moralizing Technology. Understanding and Designing the Morality of Things. Chicago and London: University of Chicago Press.

Von Schomberg, R. 2013. A Vision of Responsible Research and Innovation. In Responsible Innovation, eds. R. Owen, J. Bessant, and M. Heintz, 51-74. Chichester: Wiley. 